\title{
Preliminary Screening of Maize Accessions Against Common Rust Disease (Puccinia sorghi Schw) in Arsi Negele District, Ethiopia
}

\author{
Solomon Mengistu \\ Harar Biodiversity Center, Ethiopian Biodiversity Institute, Addis Ababa, Ethiopia
}

Email address:

mengistusolomon7@gmail.com

\section{To cite this article:}

Solomon Mengistu. Preliminary Screening of Maize Accessions Against Common Rust Disease (Puccinia sorghi Schw) in Arsi Negele District, Ethiopia. American Journal of Bioscience and Bioengineering. Vol. 9, No. 3, 2021, pp. 75-80. doi: 10.11648/j.bio.20210903.13

Received: April 23, 2021; Accepted: June 1, 2021; Published: June 22, 2021

\begin{abstract}
Maize is one of the most important crops in Ethiopia. The production of the crop is threatened by a number of biotic and a biotic factor. Among biotic factors, common rust caused by Puccinia sorghi Schw, is one of destructive foliar diseases of maize in Ethiopia. A study was conducted during the main cropping season (April to November, 2016) in the field at Arsi Negele research farm, Ethiopia with the objective of screening 92 maize accessions and 2 farmer's verities checks against common rust disease. The experiment was laid out in augmented design with no replications. Disease assessment was done as percentage of leaf area infected on individual plant at 15 days intervals starting from 55 days after planting to cropping season for 5 times. The mean values of disease incidence, severity and AUDPC were calculated and yield recorded. The higher disease percent severity and AUDPC, least yield and thousand seed weight were observed from accessions, AN-239620, AN9195, AN-9988, AN-9991, AN-15459, AN-15460, AN-16021, AN-18121, AN-18122 and Check-1 with mean values (63.94\%, $1694.5 \%$-in days, 3.04 to $3.69 \mathrm{t} /$ ha and 240 to $297 \mathrm{~g}$ ) respectively and categorized in moderately susceptible group. Conversely, the minimum percent disease severity, AUDPC, higher yield and thousand seed weight were recorded from accessions, AN16559, AN-9987, AN-16571, AN-18104, AN-18106, AN-18121, AN2-4297, AN-913, AN-15325 AN-15326, AN-15461, AN16276, AN-16278, AN-241584 and AN-162383, with mean values of (31.01\%, 720.4\%-in days, 4.81 to 5.59 t/ha and 380 to $442 \mathrm{~g}$ ) respectively and grouped in resistant category. Therefore, accessions recorded low percent disease severity and AUDPC values could be used as the resistant varieties and source of resistance to common rust for varietal development of maize.
\end{abstract}

Keywords: Maize, Accession, Puccinia sorghi, Severity, AUDPC and Resistant

\section{Introduction}

Maize (Zea mays L.) is one of the most important food crops world-wide. It is the principal component of human diet and feed constituent for domestic animals. It ranks third in production worldwide following wheat and rice and is grown in most parts of the world over a wide range of environmental conditions [1]. It occupies an important position in the world economy as food, feed, and industrial grain crop. It is a staple food for million people in the developing countries where they obtain their protein and calorie requirements from it.

In Ethiopia, maize is one of the most important cereal crops grown. Among cereal crops, maize ranks second to tef (Eragrostis tef) in area coverage but first in productivity and total production [2]. Maize is currently produced by more farmers than any other crops. Although, improved cultivars have been largely included in the national extension package, the national average yield of maize is only 3.45 tons/ha [3], which is below the world average of 5.5 tons $/$ ha.

The low yield is attributed to a number of factors such as biotic (diseases, insect pests, and weeds), a biotic (moisture, soil fertility, etc). Among biotic factors, foliar diseases such as leaf blight (Exserhilum turcicum), and common rust (Puccinia sorghi Schw) are generally among the important constraints in tropical maize production [4]. Common rust caused by Puccinia sorghi Schw is a destructive disease in the major maize growing high land areas of the country and causes from $12-61 \%$ yield loss in disease epidemic years [5].

The use of fungicide is beyond the access of resource 
constrained farmers and moreover increases the production cost, hazardous to human health and has a negative impacts to environment.

The use of resistant varieties is effective for resource constrained farmers. This is the durable and economical means for disease management. This principle is equally important for an eco-friendly environment.

Therefore, this study was carried out to investigate the potential of 92 maize accessions against common rust disease which serve as a preliminary information that could help in developing Puccinia sorghi resistant and high yielding maize varieties under field conditions.

\section{Materials and Methods}

\subsection{Description of the Study Area}

Field experiment was conducted at Negele research farm site in Arsi Negele District, West Arsi Zone, Oromia Regional State, Ethiopia during the main cropping season of 2016. It is located at $7^{\circ} 21^{\prime} \mathrm{N} 38^{\circ} 42^{\prime} \mathrm{E}$ and at an elevation of 1940 m.a s 1. It has a chromic and pellic vertisols with PH of 5-7. The annual rainfall of the location is $915 \mathrm{~mm}$ with $27 \pm 0.30^{\circ} \mathrm{C}$ mean daily temperature

\subsection{Materials Used}

Ninety two maize accessions obtained from Ethiopian Biodiversity Institute (Gene bank) and two farmer's verities check named as check 1 and 2 were sown at Arsi Negele research farm site to evaluate against common rust disease at different plant growth stages during the main cropping seasons in 2016.

\subsection{Experimental Design}

92 maize accessions without replication along with 2 replicated checks were arranged in augmented design. Individual plot size measured $9 \mathrm{~m} * 1.5 \mathrm{~m}$ with 4 maize rows planted at a spacing of $75^{*} 30 \mathrm{~cm}$. All plots were planted manually with two seeds per hole. Fertilizers (DAP \& Urea) and all agronomic practices were applied based on the recommendations.

\subsection{Inoculation and Disease Assessment}

Natural inoculation was relied up on in all experimental plots. Disease incidence and severity on 10 tagged plants was assessed on two weeks interval. Starting from the first appearance of disease symptoms (55 DAP) a total of five disease assessments were conducted throughout the cropping season. Disease assessment was made for the various accessions from the central two rows. Incidence of common rust was assessed by counting the number of plants on the middle two rows and expressed as percentage of total plants. Disease incidence was computed according to the following equation:

$$
\text { Incidence }(\%)=\frac{\text { Number of diseased plant }}{\text { Total number of plants }} * 100
$$

Disease severity (the percentage area of leaves damage per plant on 10 randomly pre-tagged maize plants in each plot) was rated following CIMMYT methods using 1-5 scoring scale [6]; where 1.0=Very slight to slight infection, one or two to few scattered lesions on lower leaves only; 2.0=Light infection, moderate number of lesions on lower leaves only, 3.0 =Moderate infection, abundant lesions are on lower leaves, few on middle leaves, $4.0=$ Heavy infection, lesions are abundant on lower and middle leaves, extending to upper leaves, 5.0=Very heavy infection, lesions abundant on almost all leaves, plants prematurely dry or killed by the disease. The severity grades were converted into percentage severity index (PSI) for analysis [7].

$$
P S I=\frac{\text { Sum of numerical rating }}{\text { No of plants observed (10)x maximum score scale(5) }} * 100
$$

The disease progress rate (r) was calculated for each plot by using the linear logistic model and the calculated value was analyzed. These parameters were used in analysis of variance to compare the disease progress among the accessions. Apparent infection rate answers the question 'does disease develop more rapidly in plot of $A$ than in plot of $\mathrm{B}^{\prime}$ [8].

$$
r=\frac{\left(\ln \frac{x}{1-x}\right)-\left(\ln \frac{x \mathrm{o}}{1-x_{0}}\right)}{t}
$$

Where, $\mathrm{r}=$ infection rate, $\mathrm{xo}=$ initial disease severity, $\mathrm{x}=$ final disease severity and $t$ is the duration of the epidemic, $\ln$ $=$ natural logarithm.

\subsection{Area Under Disease Progress Curve (AUDPC)}

To ensure disease evaluation in the field was consistent, a disease progress curve was made. By constructing a curve, symptom development and disease severity could be compared. The area under disease progress curve (AUDPC) was used to quantify disease progression from the beginning of the epidemic and the time until the rust and blight reached peak. AUDPC shows the level of disease that induces stress during the season and can be used as a predictor of yield. An area under the disease progress curve (AUDPC) was calculated for each plot using PSI values. The derived disease parameter, area under the disease progress curve (AUDPC), was calculated for each plot using the formula of [8]:

$$
\operatorname{AUDPC}_{\mathrm{i}=1}^{\mathrm{n}-1}=\Sigma[\{(\mathrm{Yi}+\mathrm{Yi}+1) / 2\} \times(\mathrm{t}(\mathrm{i}+1)-\mathrm{ti})]
$$

Where, $\mathrm{Yi}=$ disease severity on the $\mathrm{i}^{\text {th }}$ date, $\mathrm{ti}=$ time on which $\mathrm{Yi}$ was recorded and $\mathrm{n}=$ total number of observation.

\subsection{Grain Yield and Thousand Seed Weight}

At maturity, the yields of the 92 maize accessions and 2 farmer's verities checks were harvested manually from each plot. The yield from the plots was converted into tone per hectare $\left(\mathrm{t} / \mathrm{ha}^{1}\right)$. And one thousand randomly taken seeds from each plot were weighed separately and thousand seed weight was measured in gram. 


\subsection{Data Analysis}

Data on all disease parameters (disease incidence, disease severity, PSI, AUDPC and disease progression rate (r), yield and thousand seed weight were subjected to analysis of variance (ANOVA) using Statistical Analysis System version 9.1 software (SAS Institute, 2002). Duncan's multiple range test (DMRT) was used for comparison among accessions means $(\mathrm{P}<0.05)$, where there is a significant difference. Regression analysis was used to examine the relationship between severity of foliage disease (independent variables) and change in yield and thousand grain weights (dependent variables).

\section{Results and Discussion}

\subsection{Disease Incidence}

Result of disease incidence revealed that no significant differences $(\mathrm{P}<0.05)$ was observed among accessions in final per cent disease incidence (Table A1).

\subsection{Disease Severity}

There were significant differences $(\mathrm{p}<0.05)$ among maize accessions for final percent disease severity. The least percent disease severity were recorded for AN-16559, AN-9987, AN16571, AN-18104, AN-18106, AN-18121, AN2-4297, AN913, AN-15325, AN-15326, AN-15461, AN-16276, AN16278, AN-241584 and AN-162383 accessions with mean values of $31.01 \%$. Conversely, the highest per cent disease severity was recorded for AN-239620, AN-9195, AN-9988, AN-9991, AN-15459, AN-15460, AN-16021, AN-18121, AN -18122 and check-1 with mean values of $63.94 \%$. Moreover, based on the mean values of percent severity, the ninety two maize accessions and 2 checks could be categorized into three resistance groups, i.e. resistant (R), moderately resistant (MR) and moderately susceptible (MS). Among them, fifteen accessions, AN-16559, AN-9987, AN-16571, AN-18104, AN-18106, AN-18121, AN2-4297, AN-913, AN-15325, AN15326, AN-15461, AN-16276, AN-16278, AN-241584 and AN-162383 with mean values (31.01\%), were found resistant, while eight accessions, AN-16570, AN-15324, AN15466, AN-15467, AN-15462, AN-15327, AN-15455 and AN-15328,(37.13 to 44\%) were found moderately resistant (Table A1). Seventy accessions and check-2 were found moderately susceptible with mean values of (46.8 to 63.91\%). (Table A1).

Similarly, genotypes were placed in reaction groups using CIMMYT scale, where 0 were placed in immune group, 0 $10 \%$ in highly resistant group, $11-34 \%$ in resistant group, $35-$ $44 \%$ in moderately resistant group, $45-69 \%$ in moderately susceptible category and genotypes with disease severity of more than $70 \%$ were placed in susceptible group [6].

\subsection{Area Under Disease Progressive Curve (AUDPC)}

The common rust symptoms appeared to start in all plots at about the same time with uneven distribution of the pathogen. But gradually the disease severity varied within and among plots. The results of areas under disease progress curves were showed significantly different among the accessions $(\mathrm{P}<0.05)$.

The highest AUDPC were recorded for accessions, AN239620, AN-9195, AN-9988, AN-9991, AN-15459, AN15460, AN-16021, AN-18121, AN-18122 and check-1 with mean values (1694.5\%-days). Conversely, the lowest AUDPC values with mean values $(720.4 \%$ days \%-days) was observed from accessions, AN-16559, AN-9987, AN16571, AN-18104, AN-18106, AN-18121, AN-24297, AN9183, AN-15325, AN-15326, AN-15461, AN-16276, AN$16278, \mathrm{AN}-241584$ and AN-16233 with the value (Table A1)

On the other hand, the mean value of AUDPC provides adequate evaluation of the reaction of the accessions against common maize rust disease. The higher area under disease progress curves were observed on moderately susceptible accessions than resistant and moderately resistant groups.

Similarly AUDPC is directly related with the yield loss [9]. Therefore, selection of genotype having lower AUDPC is acceptable for practical purposes. Disease progress curve models also provide basic information for planning effective disease management practices for genotype with different types and levels of resistance [10].

\subsection{Disease Progressive Rate (r)}

Comparisons of the disease development rate among accessions using the logistic model showed that disease progressive rates significantly differed between accessions $(\mathrm{P}<0.05)$. The higher disease progressive rates were recorded from AN-239620, AN-9195, AN-9988, AN-9991, AN-15459, AN-15460, AN-16021, AN-18121, AN-18122 and check-1 with mean value $(0.81)$. Likewise, the lowest disease progressive rate were recorded from accessions, AN-16559, AN-9987, AN- 16571, AN-18104, AN-18106, AN-18121, AN2-4297, AN-9183, AN-15325, AN-15326, AN-15461, AN-16276, AN-16278, AN-241584 and AN-16233, with mean value $(0.33)$.

\subsection{Yield and Thousand Seed Weight}

Grain yield and thousand seed weight varied significantly among accession $(p<0.05)$. The highest grain yields of 15 maize accessions ranged from 4.81 to $5.59 \mathrm{t} / \mathrm{ha}$ and thousand seed weights of (380 to $442 \mathrm{~g}$ ) were obtained from AN16559, AN-9987, AN-16571, AN-18104, AN-18106, AN18121, AN-24297, AN-9183, AN-15325, AN-15326, AN15461, AN-16276, AN-16278, AN-241584 and AN-16233 respectively, in 2016 main cropping season. Conversely, the lowest grain yields along with thousand seed weight (3.04 to $3.69 \mathrm{t} / \mathrm{ha}$ ) and (240 to $297 \mathrm{~g}$ ) were measured on accessions, AN-239620, AN-9195, AN-9988, AN-9991, AN-15459, AN15460, AN-16021, AN-18121 and AN-18122 respectively (Table A1). Disease severity and AUDPC showed negative correlations with yield and thousand seed weight. Likewise, yield and thousand seed weight had positive correlations. 


\section{Conclusion}

Rust disease is among devastating foliar fungal diseases of maize in Ethiopia. Evaluation of maize accessions against common rust was studied at Arsi Negele using 92 maize accessions and 2 farmers' verities checks. There was significant difference among accessions against common rust disease in percent disease severity, AUDPC, infection rate, yield and thousand seed weight at Arsi Negele.

In conclusion, the maize accessions varied highly in common rust severity; screening of accessions seemed to be one of the important techniques for finding the sources of resistant. Hence, among the tested maize accessions, AN16559, AN-9987, AN-16571, AN-18104, AN-18106, AN-
18121, AN-24297, AN-9183, AN-15325, AN-15326, AN15461, AN-16276, AN-16278, AN-241584 and AN-16233, were found resistant against common rust disease.

Therefore, the present study provides crucial information in searching source of resistant varietal development and to confirm the disease reaction further investigation could be recommended.

\section{Acknowledgements}

The author of the research would like to thank the Ethiopian Biodiversity Institute for their financial support to conduct this study.

\section{Appendix}

Table A1. Final disease incidence percent, final severity percent, AUDPC percent in days, infection rate units per day, resistance category, thousand seed weight and yield per hectare in tone.

\begin{tabular}{|c|c|c|c|c|c|c|c|}
\hline $\begin{array}{l}\text { Accession } \\
\text { name }\end{array}$ & $\begin{array}{l}\text { Final disease } \\
\text { incidence }(\%)\end{array}$ & $\begin{array}{l}\text { Final severity } \\
(\%)\end{array}$ & $\begin{array}{l}\text { AUDPC (\%- } \\
\text { days) }\end{array}$ & $\begin{array}{l}\text { Infection rate } \\
\text { (units/day) }\end{array}$ & $\begin{array}{l}\text { Resistance } \\
\text { category }\end{array}$ & $\begin{array}{l}\text { Thousand kernel } \\
\text { weight (g) }\end{array}$ & Yield (t/ha) \\
\hline AN-239620 & 100 & $63.91^{\mathrm{a}}$ & $1694.5^{\mathrm{a}}$ & $0.81^{\mathrm{a}}$ & MS & $264^{\text {edcf }}$ & $3.34^{\text {edcf }}$ \\
\hline AN-9195 & 100 & $63.91^{\mathrm{a}}$ & $1694.5^{\mathrm{a}}$ & $0.81^{\mathrm{a}}$ & MS & $292^{\text {ebdcf }}$ & $3.69^{\text {ebdcf }}$ \\
\hline AN-9988 & 100 & $63.91^{\mathrm{a}}$ & $1694.5^{\mathrm{a}}$ & $0.81^{\mathrm{a}}$ & MS & $260^{\text {edf }}$ & $3.29^{\mathrm{edf}}$ \\
\hline AN-9991 & 100 & $63.91^{\mathrm{a}}$ & $1694.5^{\mathrm{a}}$ & $0.81^{\mathrm{a}}$ & MS & $252^{\text {ef }}$ & $3.19^{\mathrm{ef}}$ \\
\hline AN-15459 & 100 & $63.91^{\mathrm{a}}$ & $1694.5^{\mathrm{a}}$ & $0.81^{\mathrm{a}}$ & MS & $297^{\text {ebdacf }}$ & $3.76^{\text {ebdac }}$ \\
\hline AN-15460 & 100 & $63.91^{\mathrm{a}}$ & $1694.5^{\mathrm{a}}$ & $0.81^{\mathrm{a}}$ & MS & $296^{\text {ebdacf }}$ & $3.75^{\text {ebdac }}$ \\
\hline AN-16021 & 100 & $63.91^{\mathrm{a}}$ & $1694.5^{\mathrm{a}}$ & $0.81^{\mathrm{a}}$ & MS & $244^{\mathrm{f}}$ & $3.09^{f}$ \\
\hline AN-18121 & 100 & $63.91^{\mathrm{a}}$ & $1694.5^{\mathrm{a}}$ & $0.81^{\mathrm{a}}$ & MS & $260^{\text {edf }}$ & $3.29^{\text {edf }}$ \\
\hline AN-18122 & 100 & $63.91^{\mathrm{a}}$ & $1694.5^{\mathrm{a}}$ & $0.81^{\mathrm{a}}$ & MS & $308^{\text {ebdacf }}$ & $3.90^{\text {ebdac }}$ \\
\hline Check-1 & 100 & $63.91^{\mathrm{a}}$ & $1694.5^{\mathrm{a}}$ & $0.81^{\mathrm{a}}$ & MS & $240^{\mathrm{f}}$ & $3.04^{\mathrm{f}}$ \\
\hline AN -16262 & 100 & $62.98^{\mathrm{a}}$ & $1658.8^{\mathrm{a}}$ & $0.80^{\mathrm{a}}$ & MS & $276^{\text {edcf }}$ & $3.49^{\text {edcf }}$ \\
\hline AN-9989 & 100 & $62.98^{\mathrm{a}}$ & $1658.8^{\mathrm{a}}$ & $0.80^{\mathrm{a}}$ & MS & $276^{\text {ebdcf }}$ & $3.49^{\text {rdcf }}$ \\
\hline AN-9992 & 100 & $62.98^{\text {aa }}$ & $1658.8^{\mathrm{a}}$ & $0.80^{\mathrm{a}}$ & MS & $260^{\text {edf }}$ & $3.29^{\mathrm{edf}}$ \\
\hline AN-15456 & 100 & $62.98^{\mathrm{a}}$ & $1658.8^{\mathrm{a}}$ & $0.80^{\mathrm{a}}$ & MS & $276^{\text {ebdcf }}$ & $3.49^{\text {edcf }}$ \\
\hline AN- 15458 & 100 & $62.98^{\mathrm{a}}$ & $1658.8^{\mathrm{a}}$ & $0.80^{\mathrm{a}}$ & MS & $272^{\text {ebdcf }}$ & $3.44^{\text {edcf }}$ \\
\hline Check-2 & 100 & $61.8^{\text {ba }}$ & $1629.3^{\text {ba }}$ & $0.79^{\text {ba }}$ & MS & $269^{f}$ & $3.32^{\text {edcf }}$ \\
\hline AN-18112 & 100 & $61.52^{\text {ba }}$ & $1625.1^{\text {ba }}$ & $0.79^{\text {ba }}$ & MS & $244^{\mathrm{f}}$ & $3.09^{f}$ \\
\hline AN-9990 & 100 & $60.70^{\text {ba }}$ & $1597.6^{\text {ba }}$ & $0.72^{\text {bac }}$ & MS & $260^{\mathrm{f}}$ & $3.29^{\text {edcf }}$ \\
\hline AN-16269 & 100 & $60.70^{\text {ba }}$ & $1597.6^{\text {ba }}$ & $0.72^{\text {bac }}$ & MS & $336^{\text {ebdacf }}$ & $4.25^{\mathrm{ebdac}}$ \\
\hline AN-16563 & 100 & $59.38^{\text {ba }}$ & $1567.4^{\text {ba }}$ & $0.71^{\text {bac }}$ & MS & $260^{\text {edf }}$ & $3.29^{\text {edff }}$ \\
\hline AN-16023 & 100 & $59.38^{\text {ba }}$ & $1567.4^{\text {ba }}$ & $0.71^{\text {bac }}$ & MS & $332^{\text {ebdacf }}$ & $4.20^{\text {ebdac }}$ \\
\hline AN-16236 & 100 & $58.12^{\text {ba }}$ & $1534.9^{\text {ba }}$ & $0.64^{\mathrm{bac}}$ & MS & $344^{\text {ebdacf }}$ & $4.35^{\text {ebdac }}$ \\
\hline AN-18098 & 100 & $57.79^{\mathrm{ba}}$ & $1509.7^{\text {ba }}$ & $0.61^{\text {bac }}$ & MS & $324^{\text {ebdacf }}$ & $4.10^{\text {ebdac }}$ \\
\hline AN-9182 & 100 & $57.79^{\mathrm{ba}}$ & $1509.7^{\text {ba }}$ & $0.61^{\text {bac }}$ & MS & $320^{\text {ebdacf }}$ & $4.05^{\mathrm{ebdac}}$ \\
\hline AN-16020 & 100 & $57.74^{\text {ba }}$ & $1508.1^{\mathrm{ba}}$ & $0.61^{\text {bac }}$ & MS & $324^{\text {ebdacf }}$ & $4.10^{\text {ebdac }}$ \\
\hline AN-18108 & 100 & $57.0^{\text {ba }}$ & $1503.1^{\text {ba }}$ & $0.61^{\text {bac }}$ & MS & $372^{\text {bac }}$ & $4.71^{\mathrm{bac}}$ \\
\hline AN-9994 & 80 & $55.05^{\text {bac }}$ & $1451.3^{\mathrm{bac}}$ & $0.59^{\text {bac }}$ & MS & $380^{\text {bac }}$ & $4.81^{\mathrm{ba}}$ \\
\hline AN-9191 & 80 & $55.05^{\text {bac }}$ & $1451.3^{\mathrm{bac}}$ & $0.59^{\mathrm{bac}}$ & MS & $328^{\text {ebdacf }}$ & $4.15^{\text {ebdac }}$ \\
\hline AN-16012 & 100 & $55.05^{\text {bac }}$ & $1451.3^{\mathrm{bac}}$ & $0.59^{\mathrm{bac}}$ & MS & $348^{\text {ebdacf }}$ & $4.40^{\text {ebdac }}$ \\
\hline AN-16567 & 80 & $55.05^{\text {bac }}$ & $1451.3^{\mathrm{bac}}$ & $0.59^{\text {bac }}$ & MS & $280^{\text {edcf }}$ & $3.54^{\text {edcf }}$ \\
\hline AN-16582 & 80 & $55.05^{\text {bac }}$ & $1451.3^{\mathrm{bac}}$ & $0.59^{\text {bac }}$ & MS & $328^{\text {ebdacf }}$ & $4.15^{\text {ebdac }}$ \\
\hline AN-18096 & 80 & $55.05^{\mathrm{bac}}$ & $1451.3^{\mathrm{bac}}$ & $0.59^{\text {bac }}$ & MS & $324^{\text {ebdacf }}$ & $4.10^{\text {ebdac }}$ \\
\hline AN-237684 & 100 & $55.05^{\text {bac }}$ & $1451.3^{\mathrm{bac}}$ & $0.59^{\text {bac }}$ & MS & $312^{\text {ebdacf }}$ & $3.95^{\text {ebdac }}$ \\
\hline AN-9188 & 80 & $55.05^{\text {bac }}$ & $1451.3^{\mathrm{bac}}$ & $0.59^{\text {bac }}$ & MS & $348^{\text {ebdacf }}$ & $4.40^{\text {ebdac }}$ \\
\hline AN-9984 & 80 & $55.05^{\text {bac }}$ & $1451.3^{\mathrm{bac}}$ & $0.59^{\text {bac }}$ & MS & $332^{\text {ebdacf }}$ & $4.20^{\mathrm{ebdac}}$ \\
\hline AN-9995 & 100 & $55.05^{\text {bac }}$ & $1451.3^{\mathrm{bac}}$ & $0.59^{\text {bac }}$ & MS & $376^{\mathrm{bac}}$ & $4.76^{\text {bac }}$ \\
\hline AN-16561 & 100 & $55.05^{\text {bac }}$ & $1451.3^{\mathrm{bac}}$ & $0.59^{\text {bac }}$ & MS & $316^{\text {ebdacf }}$ & $4.00^{\text {ebdacf }}$ \\
\hline AN-237657 & 100 & $55.05^{\text {bac }}$ & $1451.3^{\mathrm{bac}}$ & $0.59^{\text {bac }}$ & MS & $376^{\text {bac }}$ & $4.76^{\mathrm{bac}}$ \\
\hline AN-18114 & 80 & $54.52^{\text {bac }}$ & $1424.0^{\mathrm{bac}}$ & $0.58^{\text {bac }}$ & MS & $360^{\text {bdac }}$ & $4.55^{\text {ebdac }}$ \\
\hline AN-16261 & 80 & $54.52^{\text {bac }}$ & $1424.0^{\mathrm{bac}}$ & $0.58^{\text {bac }}$ & MS & $316^{\text {ebdacf }}$ & $4.00^{\text {ebdacf }}$ \\
\hline
\end{tabular}




\begin{tabular}{|c|c|c|c|c|c|c|c|}
\hline $\begin{array}{l}\text { Accession } \\
\text { name }\end{array}$ & $\begin{array}{l}\text { Final disease } \\
\text { incidence }(\%)\end{array}$ & $\begin{array}{l}\text { Final severity } \\
(\%)\end{array}$ & $\begin{array}{l}\text { AUDPC (\%- } \\
\text { days) }\end{array}$ & $\begin{array}{l}\text { Infection rate } \\
\text { (units/day) }\end{array}$ & $\begin{array}{l}\text { Resistance } \\
\text { category }\end{array}$ & $\begin{array}{l}\text { Thousand kernel } \\
\text { weight (g) }\end{array}$ & Yield (t/ha) \\
\hline AN -9997 & 80 & $54.52^{\text {bac }}$ & $1424.0^{\text {bac }}$ & $0.58^{\mathrm{bac}}$ & MS & $344^{\text {ebdacf }}$ & $4.35^{\text {ebdac }}$ \\
\hline AN-16025 & 80 & $53.99^{\text {bac }}$ & $1379.1^{\text {bac }}$ & $0.56^{\mathrm{bac}}$ & MS & $316^{\text {ebdacf }}$ & $4.00^{\text {ebdacf }}$ \\
\hline AN-18103 & 100 & $53.99^{\text {bac }}$ & $1379.1^{\text {bac }}$ & $0.56^{\mathrm{bac}}$ & MS & $312^{\text {ebdacf }}$ & $3.95^{\mathrm{ebdac}}$ \\
\hline AN-237597 & 80 & $53.99^{\text {bac }}$ & $1379.1^{\text {bac }}$ & $0.56^{\mathrm{bac}}$ & MS & $316^{\text {ebdacf }}$ & $4.00^{\text {ebdacf }}$ \\
\hline AN-9192 & 80 & $53.99^{\text {bac }}$ & $1379.1^{\mathrm{bac}}$ & $0.56^{\text {bac }}$ & MS & $336^{\text {ebdacf }}$ & $4.25^{\mathrm{ebdac}}$ \\
\hline AN -9193 & 80 & $53.99^{\text {bac }}$ & $1379.1^{\text {bac }}$ & $0.56^{\mathrm{bac}}$ & MS & $276^{\text {ebdcf }}$ & $3.49^{\text {edcf }}$ \\
\hline AN -9194 & 100 & $53.99^{\text {bac }}$ & $1379.1^{\text {bac }}$ & $0.56^{\mathrm{bac}}$ & MS & $352^{\text {ebdacf }}$ & $4.45^{\text {ebdac }}$ \\
\hline AN-9998 & 80 & $53.99^{\text {bac }}$ & $1379.1^{\text {bac }}$ & $0.56^{\mathrm{bac}}$ & MS & $368^{\text {bdac }}$ & $4.66^{\text {bdac }}$ \\
\hline AN-16279 & 100 & $53.99^{\text {bac }}$ & $1379.1^{\mathrm{bac}}$ & $0.56^{\mathrm{bac}}$ & MS & $372^{\text {bac }}$ & $4.71^{\mathrm{bac}}$ \\
\hline AN-9993 & 100 & $52.13^{\text {bac }}$ & $1353.3^{\text {bac }}$ & $0.54^{\mathrm{bac}}$ & MS & $332^{\text {ebdacf }}$ & $4.20^{\mathrm{ebdac}}$ \\
\hline AN-16226 & 80 & $52.13^{\text {bac }}$ & $1353.3^{\text {bac }}$ & $0.54^{\mathrm{bac}}$ & MS & $368^{\text {bdac }}$ & $4.66^{\mathrm{bdac}}$ \\
\hline AN-18100 & 100 & $52.13^{\text {bac }}$ & $1353.3^{\mathrm{bac}}$ & $0.54^{\mathrm{bac}}$ & MS & $328^{\text {ebdacf }}$ & $4.15^{\mathrm{ebdac}}$ \\
\hline AN-228786 & 80 & $52.13^{\mathrm{bac}}$ & $1353.3^{\text {bac }}$ & $0.54^{\mathrm{bac}}$ & MS & $348^{\text {ebdacf }}$ & $4.40^{\text {ebdac }}$ \\
\hline AN-9181 & 100 & $52.13^{\text {bac }}$ & $1353.3^{\text {bac }}$ & $0.54^{\mathrm{bac}}$ & MS & $328^{\text {ebdacf }}$ & $4.15^{\mathrm{ebdac}}$ \\
\hline AN-15247 & 80 & $52.13^{\text {bac }}$ & $1353.3^{\text {bac }}$ & $0.54^{\mathrm{bac}}$ & MS & $320^{\text {ebdacf }}$ & $4.0^{\mathrm{ebdac}}$ \\
\hline AN -9187 & 100 & $51.65^{\text {bac }}$ & $1330.3^{\text {bac }}$ & $0.53^{\mathrm{bac}}$ & MS & $330^{\text {ebdacf }}$ & $4.18^{\text {ebdac }}$ \\
\hline AN - 16234 & 80 & $51.65^{\mathrm{bac}}$ & $1330.3^{\text {bac }}$ & $0.53^{\text {bac }}$ & MS & $360^{\text {bdac }}$ & $4.55^{\mathrm{ebdac}}$ \\
\hline AN -98098 & 80 & $51.65^{\mathrm{bac}}$ & $1330.3^{\text {bac }}$ & $0.53^{\text {bac }}$ & MS & $352^{\text {ebdacf }}$ & $4.45^{\mathrm{ebdac}}$ \\
\hline AN -9985 & 80 & $51.65^{\text {bac }}$ & $1330.3^{\text {bac }}$ & $0.53^{\text {bac }}$ & MS & $328^{\text {ebdacf }}$ & $4.15^{\mathrm{ebdac}}$ \\
\hline AN-239668 & 80 & $51.65^{\text {bac }}$ & $1330.3^{\text {bac }}$ & $0.53^{\text {bac }}$ & MS & $324^{\text {ebdacf }}$ & $4.10^{\text {ebdac }}$ \\
\hline AN-15457 & 80 & $51.65^{\text {bac }}$ & $1330.3^{\text {bac }}$ & $0.53^{\text {bac }}$ & MS & $288^{\text {edcf }}$ & $3.64^{\text {edcf }}$ \\
\hline AN -15463 & 80 & $51.65^{\mathrm{bac}}$ & $1330.3^{\text {bac }}$ & $0.53^{\text {bac }}$ & MS & $344^{\text {ebdacf }}$ & $4.35^{\text {ebdac }}$ \\
\hline AN-9996 & 80 & $51.65^{\text {bac }}$ & $1330.3^{\text {bac }}$ & $0.53^{\text {bac }}$ & MS & $376^{\text {bac }}$ & $4.76^{\text {bac }}$ \\
\hline AN-239645 & 80 & $50.63^{\text {bac }}$ & $1303.8^{\text {bac }}$ & $0.51^{\mathrm{bac}}$ & MS & $356^{\text {ebdacf }}$ & $4.50^{\text {ebdcf }}$ \\
\hline AN-18113 & 80 & $50.09^{\text {bac }}$ & $1301.2^{\mathrm{bac}}$ & $0.51^{\text {bac }}$ & MS & $388^{\text {bac }}$ & $4.91^{\text {ba }}$ \\
\hline AN-10000 & 80 & $49.12^{\mathrm{bac}}$ & $1268.8^{\mathrm{bac}}$ & $0.51^{\text {bac }}$ & MS & $320^{\text {ebdacf }}$ & $4.05^{\mathrm{ba}}$ \\
\hline AN-9986 & 80 & $49.12^{\mathrm{bac}}$ & $1268.8^{\mathrm{bac}}$ & $0.51^{\text {bac }}$ & MS & $328^{\text {ebdacf }}$ & $4.15^{\mathrm{ebdac}}$ \\
\hline AN-9999 & 80 & $48.81^{\mathrm{bac}}$ & $1241.7^{\text {bac }}$ & $0.50^{\text {bac }}$ & MS & $380^{\text {bac }}$ & $4.81^{\text {bac }}$ \\
\hline AN-16562 & 80 & $48.81^{\mathrm{bac}}$ & $1241.7^{\mathrm{bac}}$ & $0.50^{\mathrm{bac}}$ & MS & $316^{\text {ebdacf }}$ & $4.00^{\text {ebdacf }}$ \\
\hline AN-9190 & 80 & $47.8^{\mathrm{bac}}$ & $1199.4^{\text {bac }}$ & $0.50^{\text {bac }}$ & MS & $340^{\text {ebdacf }}$ & $4.30^{\mathrm{ebdac}}$ \\
\hline AN-241616 & 80 & $46.8^{\mathrm{bac}}$ & $1183.0^{\text {bac }}$ & $0.49^{\text {bac }}$ & MS & $348^{\text {ebdacf }}$ & $4.40^{\text {ebdac }}$ \\
\hline AN-16570 & 80 & $44.0^{\text {bac }}$ & $1116.8^{\text {bac }}$ & $0.49^{\mathrm{bc}}$ & MR & $386^{\text {bac }}$ & $4.88^{\text {ba }}$ \\
\hline AN-15324 & 80 & $42.8^{\mathrm{bac}}$ & $1061.1^{\mathrm{bac}}$ & $0.47^{\mathrm{bc}}$ & MR & $336^{\text {ebdacf }}$ & $4.25^{\mathrm{ebdac}}$ \\
\hline AN-15466 & 80 & $42.8^{\mathrm{bac}}$ & $1061.1^{\mathrm{bac}}$ & $0.47^{\mathrm{bc}}$ & MR & $386^{\mathrm{bac}}$ & $4.88^{\mathrm{ba}}$ \\
\hline AN-15467 & 80 & $41.99^{\text {bac }}$ & $1032.3^{\text {bac }}$ & $0.46^{\mathrm{bc}}$ & MR & $376^{\text {bac }}$ & $4.76^{\text {bac }}$ \\
\hline AN-15462 & 80 & $39.61^{\text {bac }}$ & $972.3^{\text {bac }}$ & $0.44^{\mathrm{bc}}$ & MR & $364^{\text {bdac }}$ & $4.61^{\text {bdac }}$ \\
\hline AN-15327 & 80 & $38.27^{\mathrm{bac}}$ & $928.0^{\text {bac }}$ & $0.43^{\mathrm{bc}}$ & MR & $364^{\text {bdac }}$ & $4.61^{\text {bdac }}$ \\
\hline AN-15455 & 80 & $38.27^{\text {bac }}$ & $928.0^{\text {bca }}$ & $0.43^{\mathrm{bc}}$ & MR & $348^{\text {ebdacf }}$ & $4.40^{\text {ebdac }}$ \\
\hline AN-15328 & 80 & $37.13^{\mathrm{bac}}$ & $930.2^{\text {bac }}$ & $0.43^{\mathrm{bc}}$ & MR & $340^{\text {ebdacf }}$ & $4.30^{\text {ebdac }}$ \\
\hline AN-16559 & 60 & $31.01^{\mathrm{c}}$ & $720.4^{c}$ & $0.33^{\mathrm{c}}$ & $\mathrm{R}$ & $380^{\mathrm{bac}}$ & $4.81^{\text {bac }}$ \\
\hline AN-9987 & 60 & $31.01^{\mathrm{c}}$ & $720.4^{c}$ & $0.33^{\mathrm{c}}$ & $\mathrm{R}$ & $380^{\text {bac }}$ & $4.81^{\text {bac }}$ \\
\hline AN-16571 & 60 & $31.01^{\mathrm{c}}$ & $720.4^{c}$ & $0.33^{\mathrm{c}}$ & $\mathrm{R}$ & $396^{\text {ba }}$ & $5.01^{\mathrm{a}}$ \\
\hline AN-18104 & 60 & $31.01^{\mathrm{c}}$ & $720.4^{c}$ & $0.33^{\mathrm{c}}$ & $\mathrm{R}$ & $400^{\mathrm{a}}$ & $5.06^{\mathrm{a}}$ \\
\hline AN-18106 & 80 & $31.01^{\mathrm{c}}$ & $720.4^{c}$ & $0.33^{\mathrm{c}}$ & $\mathrm{R}$ & $396^{\text {ba }}$ & $5.01^{\mathrm{a}}$ \\
\hline AN-18121 & 60 & $31.01^{\mathrm{c}}$ & $720.4^{c}$ & $0.33^{\mathrm{c}}$ & $\mathrm{R}$ & $400^{\mathrm{a}}$ & $5.06^{\mathrm{a}}$ \\
\hline AN-24297 & 60 & $31.01^{\mathrm{c}}$ & $720.4^{c}$ & $0.33^{\mathrm{c}}$ & $\mathrm{R}$ & $408^{\mathrm{a}}$ & $5.16^{\mathrm{a}}$ \\
\hline AN-9183 & 60 & $31.01^{\mathrm{c}}$ & $720.4^{c}$ & $0.33^{\mathrm{c}}$ & $\mathrm{R}$ & $408^{\mathrm{a}}$ & $5.16^{\mathrm{a}}$ \\
\hline AN-15325 & 60 & $31.01^{\mathrm{c}}$ & $720.4^{c}$ & $0.33^{\mathrm{c}}$ & $\mathrm{R}$ & $420^{\mathrm{a}}$ & $5.31^{\mathrm{a}}$ \\
\hline AN-15326 & 60 & $31.01^{\mathrm{c}}$ & $720.4^{c}$ & $0.33^{\mathrm{c}}$ & $\mathrm{R}$ & $442^{\mathrm{a}}$ & $5.59^{\mathrm{a}}$ \\
\hline AN - 15461 & 60 & $31.02^{\mathrm{c}}$ & $720.4^{c}$ & $0.33^{\mathrm{c}}$ & $\mathrm{R}$ & $404^{\mathrm{a}}$ & $5.11^{\mathrm{a}}$ \\
\hline AN-16276 & 60 & $31.01^{\mathrm{c}}$ & $720.4^{c}$ & $0.33^{\mathrm{c}}$ & $\mathrm{R}$ & $408^{\mathrm{a}}$ & $5.16^{\mathrm{a}}$ \\
\hline AN-16278 & 60 & $31.01^{\mathrm{c}}$ & $720.4^{c}$ & $0.33^{\mathrm{c}}$ & $\mathrm{R}$ & $396^{\mathrm{ba}}$ & $5.01^{\mathrm{a}}$ \\
\hline AN-241584 & 60 & $31.01^{\mathrm{c}}$ & $720.4^{\mathrm{c}}$ & $0.33^{\mathrm{c}}$ & $\mathrm{R}$ & $408_{a}$ & $5.16^{\mathrm{a}}$ \\
\hline AN-16233 & 60 & $31.01^{\mathrm{c}}$ & $720.4^{c}$ & $0.33^{\mathrm{c}}$ & $\mathrm{R}$ & 400 & $5.06^{\mathrm{a}}$ \\
\hline Mean & & 52.01 & 1343 & 0.60 & & 324.67 & 4.11 \\
\hline $\mathrm{CV}$ & & 16.69 & 19.14 & 16.98 & & 11.33 & 11.31 \\
\hline $\operatorname{LSD}(5 \%)$ & $\mathrm{ns}$ & 1.26 & 1.26 & & & 1.26 & 1.26 \\
\hline
\end{tabular}

ns: none significant, CV: Coefficient of variation, LSD: Least significant difference, Means followed by the same letter in a column are not significantly different by DMRT at $5 \%$ level of significance,

AUDPC: Area Under Disease Progress Curve, MS: Moderately Susceptible; MR: Moderately Resistant and R: Resistant 


\section{References}

[1] FAOSTAT, Food and Agriculture Organization Statistics, 2012. Statistical Database of the Food and Agriculture Organization of the United Nations. http://www.fao.org [Online].

[2] CSA (Central Statistics Agency). 2014. Agricultural Sample Survey 2013/2014 (2006 E. C.). Volume I. Report on Area and Production of Major Crops (Private Peasant Holdings, Meher Season). Statistical Bulletin 532, Addis Ababa.

[3] CSA (Central Statistics Agency), 2015. Agriculture sample survey report on area and production for major crops for 2014/015. The FDRE Statistical Bulletin, CSA, Addis Ababa, Ethiopia.

[4] Renfro, B. L., Ullstrup, A. J, 1996, Comparison of maize disease in temperate and tropical environments. PANS 22: 491-498.

[5] Dey, U, Harlapur, S. I, Dhutraj D. N, Suryawanshi, A. P, Badgujar S. L, et al. 2012. Spatiotemporal yield loss assessment in corn due to common rust caused by Puccinia sorghi Schw. Afr, J. Agric Res 7 (37): 5265-5269.

[6] CIMMYT (International Maize and Wheat Improvement Center), 1985. Managing trials and reporting data for CIMMYT's international maize testing program. Mexico (DF): CIMMYT. pp. 13-14.

[7] Wheeler, J. B. E. J, 1969. An Introduction to Plant Diseases. Wiley, London, 347pp.

[8] Campbell, C. L. and Madden, L. V. 1990. Introduction to Plant Disease Epidemiology. John Wiley and Sons, New York. 531 pp.

[9] Singh, H. and Rao, M. V. 1989. Area under the disease progress curve: Its reliability as a measure of slow rusting resistance. Plant Breeding 103: 319-323.

[10] Jeger, M. J. 1993. Analyzing epidemics in time and space. Plant Pathology 32: 5-11. 